\title{
BAL QSOs in the eigenvector 1 context: toward a self-consistent model of the line absorbing/emitting regions
}

\author{
D. Dultzin-Hacyan ${ }^{1}$, P. Marziani ${ }^{2}$, J. W. Sulentic ${ }^{3}$ and C. Bongardo ${ }^{2}$ \\ ${ }^{1}$ Instituto de Astronomía, UNAM, México email: deborah@astroscu.unam.mx \\ ${ }^{2}$ INAF, Osservatorio Astronomico di Padova, Padova, Italy \\ e-mail: marziani,bongardo@pd.astro.it \\ ${ }^{3}$ Department of Physics \& Astronomy, University of Alabama, Tuscaloosa, AL, USA \\ email: giacomo@merlot.astr.ua.edu
}

\begin{abstract}
We attempt to define the structural properties of Broad Absorption Line (BAL) QSOs and their relationship with the general quasar population using the Eigenvector 1 correlations. We identify 8 low-redshift quasars $\left(\mathrm{z}<0.5,6\right.$ with a BALnicity index $>0 \mathrm{~km} \mathrm{~s}^{-1}$ ) where it was possible to combine optical and UV spectroscopic observations. The special utility of low- $z$ quasars involves our ability to discuss CIV $\lambda 1549$ BAL QSOs in the context of the Eigenvector 1 optical parameter space and to have a reliable measure of the quasar rest frame. We find that the majority of the BAL sources are population A sources as defined in Sulentic et al. (2000). At least 2 sources that are hosted by ultra-luminous IR galaxies show intriguing effects in their nuclear spectra. A possible correlation between the terminal velocity and luminosity $\mathrm{L}$ also suggests that the luminosity to black hole mass ratio $(L / M)$ is a governing factor with the largest terminal velocity BALs showing the highest $L / M$ values. The CIV $\lambda 1549$ emission line profiles of classical BALs show the ubiquitous E1 population A blue-shift that supports a disk+wind scenario with an opening angle of $<45^{\circ}$. Observation of "secondary" BAL features roughly in correspondence with the mean radial velocity of the CIV $\lambda 1549$ emission motivates us to model the BAL systems with an additional component that may share the BLR outflow and may be co-axial with the accretion disk, perhaps associated to a significant black hole spin.
\end{abstract}

\section{Introduction}

The nature of the Broad Absorption Line (BAL) QSOs remains highly debated to date, even after the discovery of many new sources through the Sloan Digital Sky Survey. At low $z$ there is a problem of rarity and identification, since finding low- $z$ BAL QSOs requires space-based observations. Only eight bright sources $m_{\mathrm{V}}<16.3$ within the redshift limit of $z<0.5$ show BALs with BALnicity Index (see Weymann et al. 1991 for the definition) larger than $0 \mathrm{~km} \mathrm{~s}^{-1}$ or a shallow but very broad absorption trough. Nonetheless, low- $z$ BAL sources provide several advantages. First, the ability to know accurately the quasar rest frame from narrow lines provides valuable constraints on geometrical and kinematical models. In addition, the so-called "Eigenvector 1" correlations have now been established as a new tool to systematise the "type 1" Active Galactic Nuclei diversity. Especially valuable is the so-called optical E1 plane defined by the FWHM of the broad component of $\mathrm{H} \beta$ and $R_{\mathrm{FeII}}=\mathrm{W}(\mathrm{FeII}$ complex at $4570 \AA) / \mathrm{W}(\mathrm{H} \beta)$ which allows us to roughly constrain the Eddington ratio and the orientation of low- $z$ quasars. A correlation between terminal velocity and luminosity $L$ already suggests that the luminosity to black hole mass ratio $(L / M)$ is a governing factor with the largest terminal velocity BALs showing the highest $L / M$ values (Marziani et al. 2003), but what is the relationship between the broad emission line region (BLR), BAL region, and the QSO inner structure? 

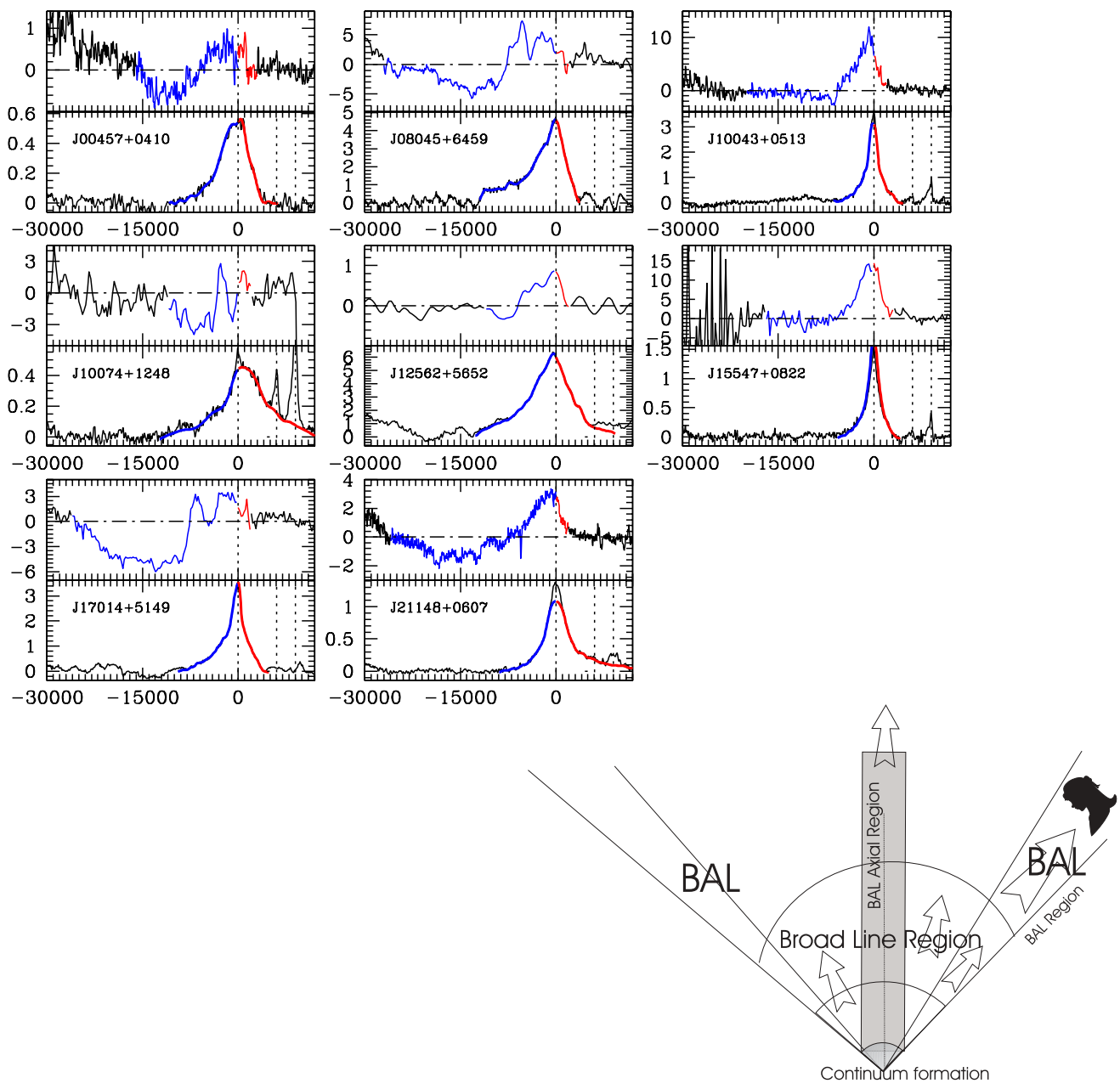

Figure 1. Upper left: CIV $\lambda 1549$ and $\mathrm{H} \beta$ inter-profile comparison after continuum and FeII subtraction. The abscissa is radial velocity from the quasar rest frame as identified by the narrow component of $\mathrm{H} \beta$ and by the [OIII] $\lambda \lambda 4959,5007$ lines. Lower right: a sketch depicting the geometry of the main components in extreme BAL QSOs like PG $1700+518$ or Mkn 231.

\section{Main results}

Systematic CIV $\lambda 1549$ blueshift: in the BAL QSOs of our sample, the CIV $\lambda 1549$ emission profile is almost completely blue-shifted with respect to the rest frame set by optical narrow lines (see Fig. 1). The blue-shift can be accounted for if the flow is predominantly radial, and if the opening angle of the flow is $<90^{\circ}$. The CIV $\lambda 1549$ profile shows a secondary, narrower absorption roughly in the middle of the emission component of several sources. The CIV $\lambda 1549$ absorption and emission profile is inconsistent with CIV $\lambda 1549_{\text {abs }}$ originating within the BLR. Acceleration should be occurring also beyond the BLR, in agreement with the hypothesis of dominating radiative forces.

The E1 plane: the data point distribution for low- $z$ quasars in the plane $\mathrm{FWHM}(\mathrm{H} \beta)$ vs. $R_{\text {Feii }}$ is essentially a sequence of Eddington ratio, with orientation acting as the major source of scatter for a narrow black hole mass range (Marziani et al. 2001). Classical BAL QSOs tend to occupy the region of higher inclination $\left(\sim 45^{\circ}\right)$. In other words, the location in the E1 optical plane of low- $z$ BAL QSOs suggests that they are misaligned 
objects, observed at an angle far from pole with respect to the accretion disk axis. Two FIR excess BAL QSOs (IRAS $0759+6508$ and Mkn 231) are outliers in the E1 optical plane, i.e. they tend to be located outside the area of major occupation in the E1 optical plane. Both sources show an impressive blue-ward asymmetry in the $\mathrm{H} \beta$ profile. Their $\mathrm{H} \beta$ emission may be additionally broadened by a circumnuclear starburst which gives rise to a Balmer-line emitting wind, or more likely, some $\mathrm{H} \beta$ emission may come from the AGN high-ionisation wind. If we use the $\operatorname{FWHM}(\mathrm{FeII})$ instead of $\operatorname{FWHM}(\mathrm{H} \beta)$ the outliers will move into the area occupied by the other classical BAL QSOs. They are actually extreme sources in terms of FeII prominence. Such extreme BAL QSOs and others with large terminal velocity seem to radiate at high Eddington ratio.

The high $z$ connection: In all the BAL QSOs of our sample, the CIV $\lambda 1549$ emission profile is almost completely blue-shifted with respect to the rest frame set by optical narrow lines. A similar condition holds also for the few high- $z$ BAL QSOs for which the rest frame is known with reasonable accuracy (just 8 sources): strong FeII emitters show CIV $\lambda 1549$ shifts that are in the thousands $\mathrm{km} \mathrm{s}^{-1}$. Note that some high- $z$ BAL QSOs studied by Korista et al. (1993) also display the double absorption structure visible in 3 of our low- $z$ sources

\section{A tentative model and conclusions}

The right panel of Fig. 1 shows a cartoon depicting the basic structure needed to account for the BAL profiles. Closer to the boundaries of the flow and beyond the BLR proper extends the BAL region in a conical corona of divergence angle $\sim 10^{\circ}$, which includes the direction of our line of sight. The observer looking at the edge of a cone of opening angle $\sim 100^{\circ}$ sees a fully blue-shifted emission component, a BAL, and a second narrower BAL originating in an axial sheet of gas participating to the general high-ionisation BLR outflow. Two-fluid models for relativistic outflows include a fast, relativistic beam surrounded by a slower, possibly thermal outflow, with a mixing layer forming between the beam and the jet (e.g. Lobanov \& Roland 2005) which could be responsible for the second absorption. This second absorption may be observable only if the black hole has a significant spin.

Our tentative conclusion is that most classical radio quiet BAL QSOs seem to be the side-view of some AGN radiating at large Eddington ratio. At low and high- $z$ we may have extreme sources - some of them may be just emerging from a cocoon of embedding accreting material, surrounded by a powerful starburst. Are BAL QSOs in a particular evolutionary stage? FIR-excess outliers most probably are. High- $z$ BAL QSOs seems to have plenty of accreting material, and at least some of them may be very similar to the local ultra-luminous FIR BAL QSOs.

\section{References}

Korista, K., et al., 1993, ApJS, 88, 357

Lobanov, A. P., Roland, J., 2005, A\&A, 431, 831

Marziani, P., et al., 2003, in: S. Collin, F. Combes \& I. Shlosman (eds.), Active Galactic Nuclei: from Central Engine to Host Galaxy, Asp Conference Series, 290, 231

Marziani, P., Sulentic, J. W., Zwitter, T., Dultzin-Hacyan D., Calvani, M., 2001, ApJ, 558, 553

Sulentic, J. W., Marziani, P., Dultzin-Hacyan, D., 2000, ARA\&A, 38, 521

Weymann, R., et al., 1991, ApJ, 373, 23 